\title{
The impact of lymph node metastases on Outcome in Uterine carcinosarcoma
}

\author{
YK Chik, KM Cheung, CH Chow, WL Leung, KH Wong, KC Ngan, Queen Elizabeth Hospital, Hong Kong
}

Background:

Uterine carcinosarcomas (UCS) are dedifferentiated (metaplastic) carcinomas comprised of carcinomatous and sarcomatous elements arising from a single malignant clone. Lymph node burden has prognostic impact on Uterine Carcinosarcoma. Lymphadenectomy is considered a standard part of surgical treatment, which was shown to improve overall survival. The median Survival with and without lymphadenectomy was shown to be 54 and 25 months, respectively. There has been recent interest in using lymph node ratio (LNR) as a prognostic tool in nodepositive endometrial carcinoma. LNR is defined as the ratio of number of positive lymph nodes to total number of lymph nodes sampled. LNR gives information on quality of surgery as well as disease burden. Previous studies have found that a high LNR score to be associated with worse progression-free survival (PFS) and overall survival (OS) in endometrial carcinoma. This is the first reported study to review the impact of LNR on uterine carcinosarcoma. the aim of this study is To determine the prognostic significance of (LNR), in patients with UCS.

Method:

We performed a retrospective review on patients diagnosed with UCS treated with total abdominal hysterectomy (+/- pelvic lymphadenectomy) with or without adjuvant treatment at Queen Elizabeth Hospital Hong Kong between 2003 to 2016. The optimal cut-off value for LNR was calculated by receiver operating characteristic $(\mathrm{ROC})$ curves. Univariate and multivariate analysis were performed to confirm the predictive value of the chosen value and to identify additional prognostic factors on overall survival (OS) and relapse-free survival (RFS).
Results:

A total of 52 patients were included, the median age were 62 (range 23-91). The median follow-up duration was 51.8 months. 22 (42.3\%), 4 (7.7\%), 18(34.6\%), 8 (15.4\%) patients had Stage I, II, III, IV disease respectively, with a 4 -year OS of $57 \%, 100 \%, 46 \%$ and $15 \%$ respectively $(p=0.006$, Figure 1). Lymphadenectomy was performed in $32(61.5 \%)$ patients. The median number of total LN sampled was 29 (range 4-54) and the median number of positive LN was 2 (range 1-11).

ROC curves showed that LNR correlates with OS and RFS $(p=0.007$ and 0.012 respectively, Figures $2 \mathrm{a} \& 2 \mathrm{~b}$ ). Those with LNR of $<3 \%$ and $>=3 \%$ had 4 -year OS of $84.4 \%$ and $24.4 \%$ respectively ( $p=0.001$, figure $3 a)$, the 4 -year RFS were $75 \%$ and $13.7 \%$ ( $p=0.016$, figure $3 b$ ) respectively. For $\mathrm{LN}$ positive patients, those with $>=3 \mathrm{LNs}$ positive were associated with worse RFS ( $p=0.055)$. Each additional positive $L N$ yielded $25 \%$ increase in relative risk of relapse.

Univariate analysis showed that adjuvant RT improves both OS and RFS ( $p=0.017$ and 0.03 respectively, figures $4 a \& 4 b$.). Multivariate analysis showed that OS and RFS were consistently improved by lymphadenectomy [adjusted hazard ratio (aHR) $0.152, p=0.003$ and aHR 0.173, $p=0.001]$. FIGO stage was associated with worse OS $(p=0.009)$. Adjuvant RT was associated with improved RFS (HR 0.281, $\mathrm{p}=0.01)$

Outcomes of different adjuvant treatments were analysed but significance of results were limited by the small sample size. For patients with stage III disease given adjuvant chemoradiation therapy $(n=4)$, survival was excellent and all patients remained disease-free up to the date of this study (follow-up duration was 21.2-51.8 months).

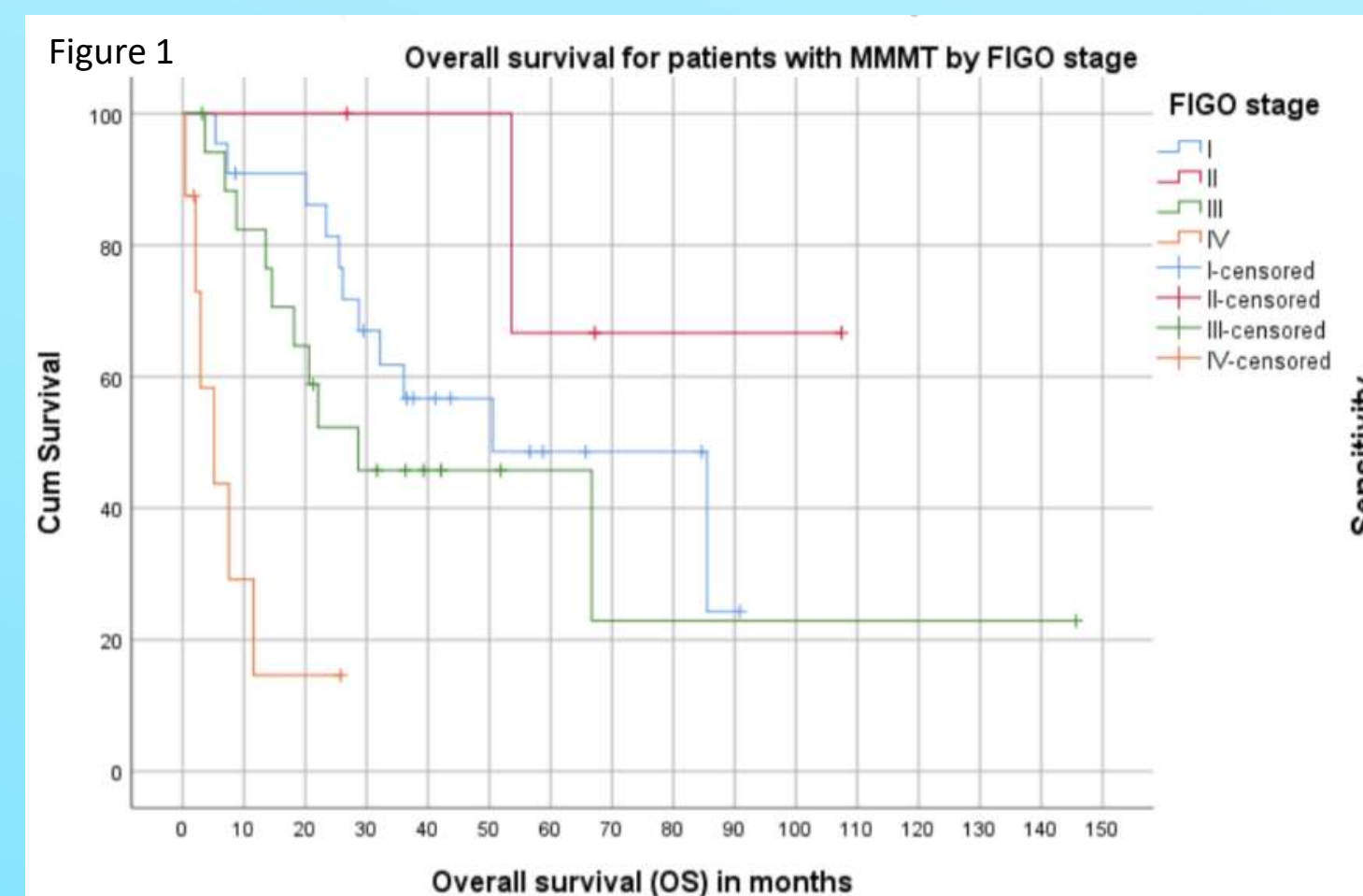

Figure 4a RFS of Stage I-IV Uterine carcinosarcoma patients given adjuvant radiotherapy

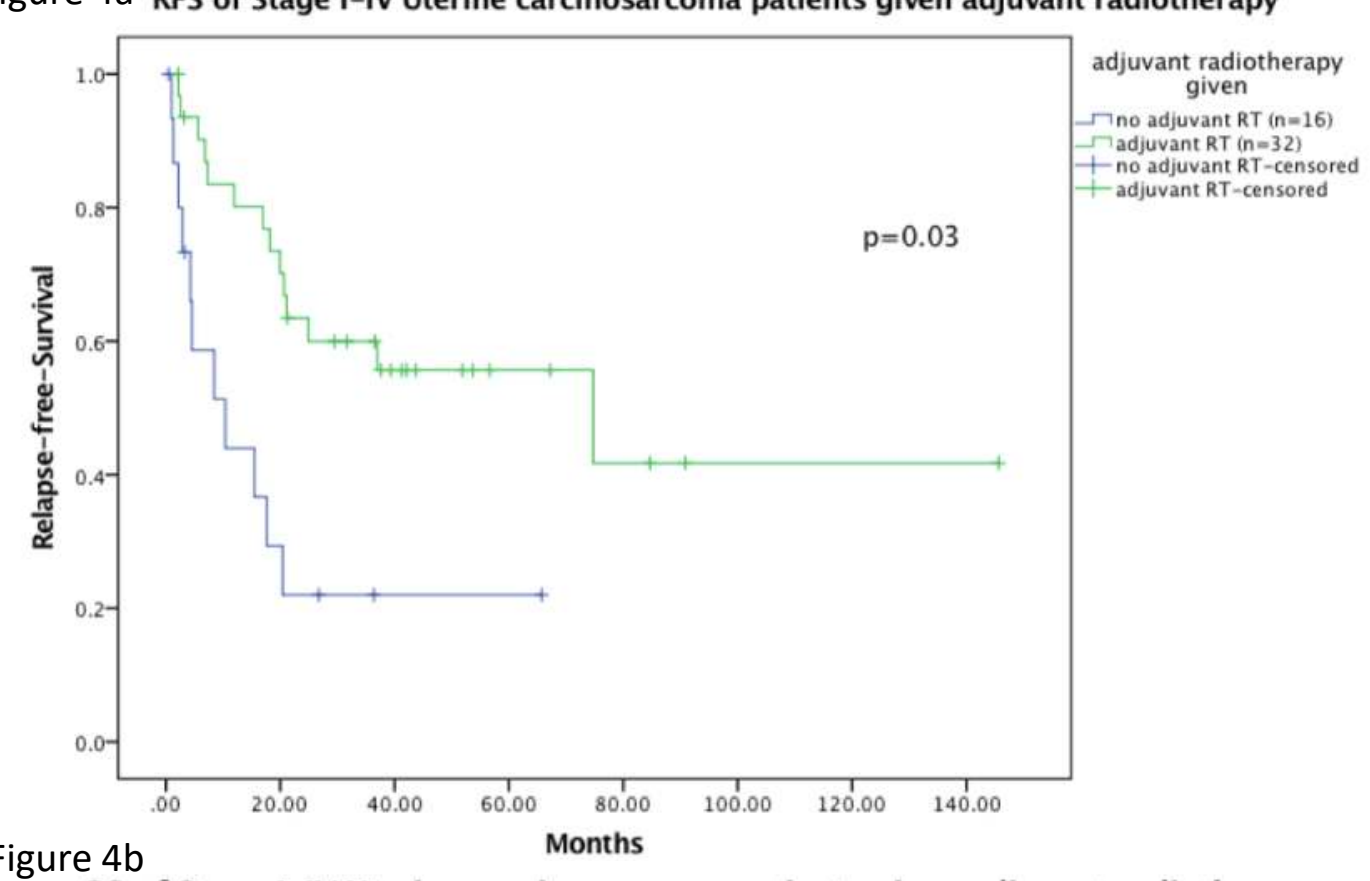

OS of Stage I-IV Uterine carcinosarcoma patients given adjuvant radiotherapy
OS

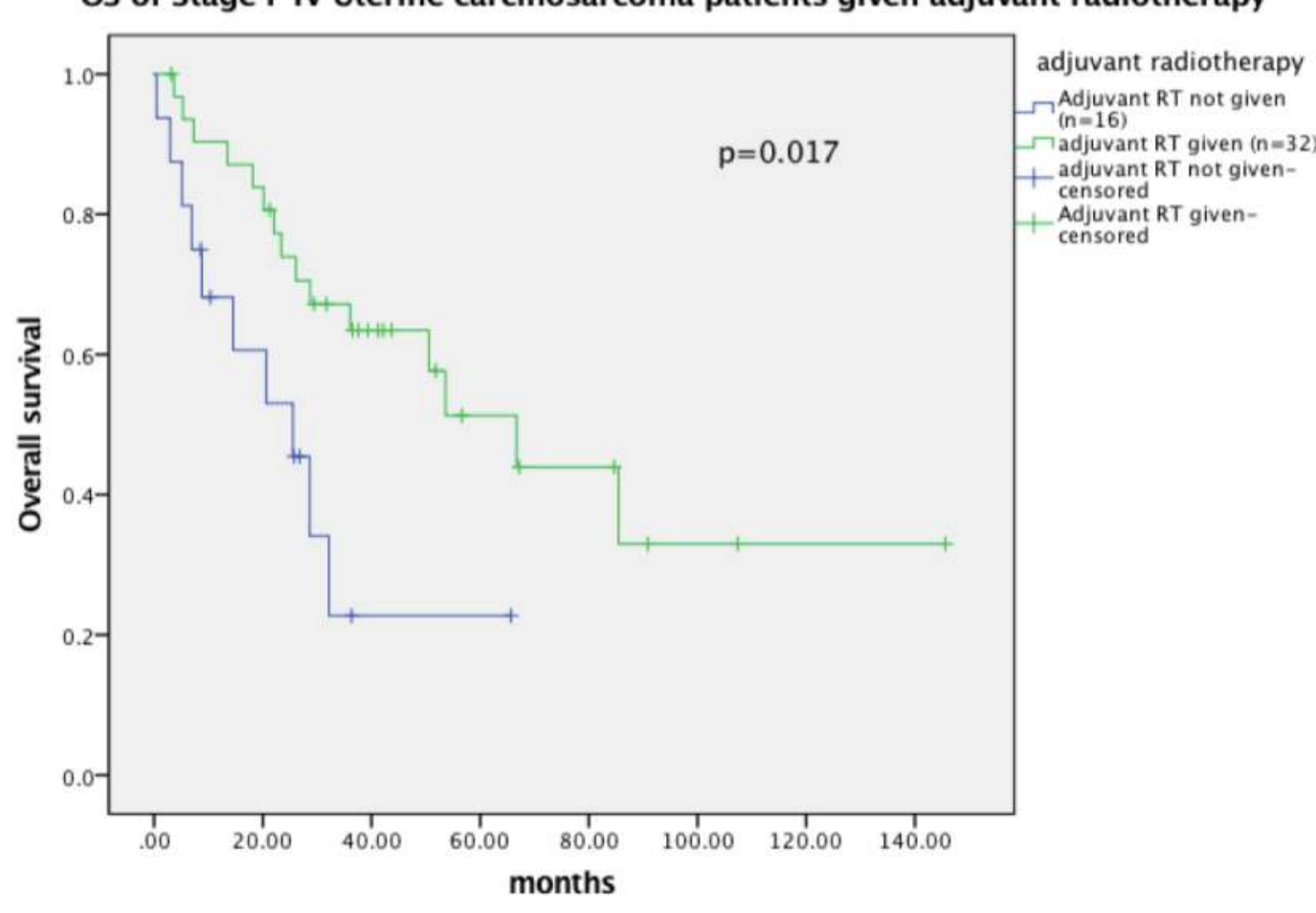

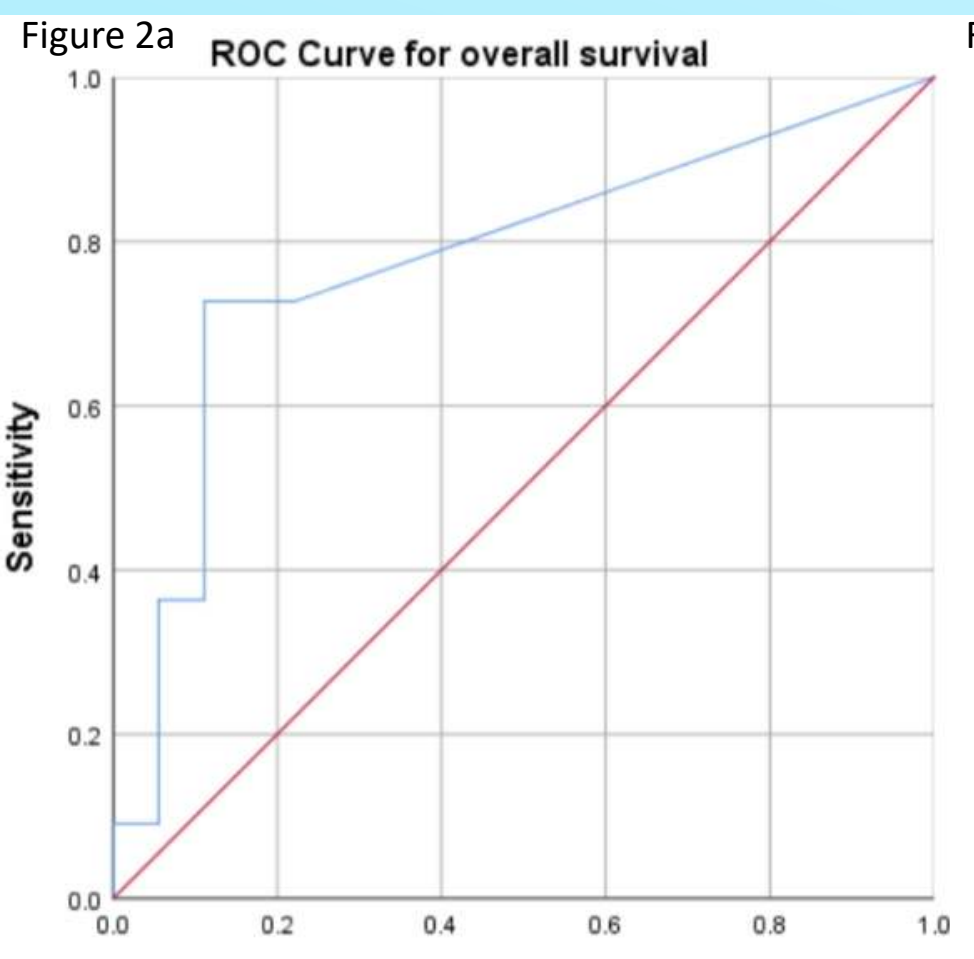

1 - Specificity
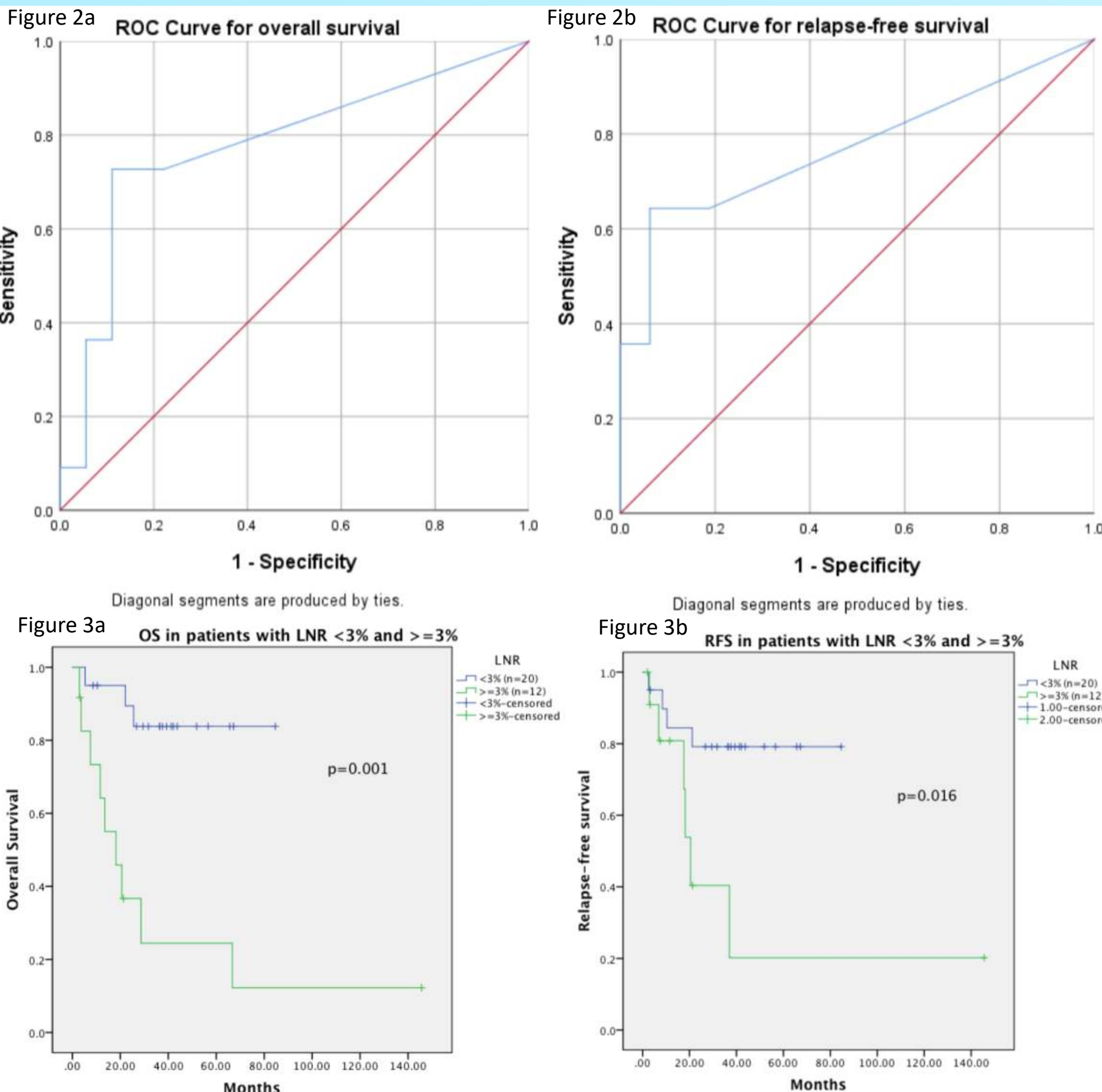

1 - Specificity

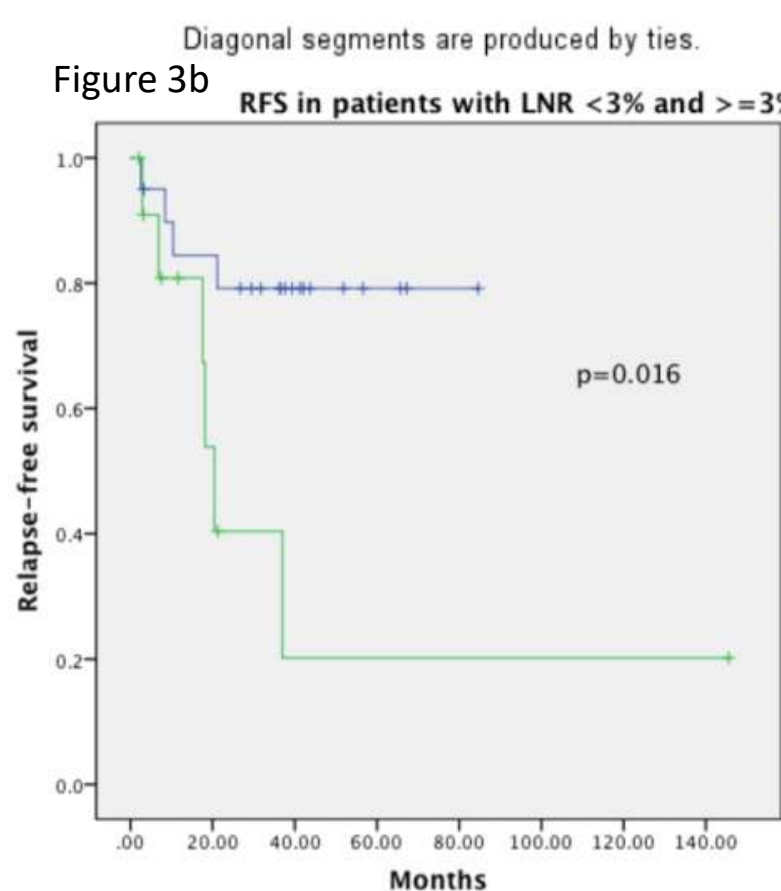

Conclusion:

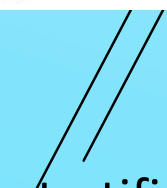

The number of positive LN and LNR are useful in prognostic stratification in UCS patients with stage III-IVa disease and may allow risk stratification of patients for intensification of adjuvant treatment. Patient with stage In disease who received adjuvant chemotherapy and radiotherapy had excellent/survival outcomes compared with historical cohort, suggesting the potential benefit of more aggressive treatment in this group of patients. 